TECHNICAL NOTE

L. Josey

A. Al Sayyari

R. Buckley

A. Coulthard

\section{Usefulness of Susceptibility-Weighted Imaging for Voxel Placement in MR Spectroscopy}

SUMMARY: MR spectroscopy is used to provide in vivo biochemical information about cerebra metabolites. Magnetic field homogeneity secondary to anatomic interfaces, hemorrhage, or necrosis may lead to suboptimal MR spectroscopy. Susceptibility-weighted imaging (SWI) can identify field inhomogeneity and could be used to guide MR spectroscopy voxel placement, leading to higher-quality MR spectroscopy examinations.
$\mathbf{P}$ roton MR spectroscopy is used in clinical and research MR imaging studies to help differentiate between regions of normal and abnormal brain tissue and to monitor disease progression. Usually, key cerebral metabolites such as $\mathrm{N}$-acetylaspartate (NAA), choline-containing compounds (Cho), and creatine/phosphocreatine $(\mathrm{Cr})$ are identified, and quantitative data or metabolite ratios are measured. ${ }^{1} \mathrm{~A}$ homogeneous magnetic field and good signal-to-noise ratio are required for high-quality spectra. Spectral quality may be compromised at both 1.5 and $3 \mathrm{~T}$ by signal-to-noise ratio issues and by magnetic field inhomogeneity, leading to poor water suppression, increased spectral line widths, and excessive baseline roll. ${ }^{2,3}$ Interfaces between bone, air, and soft tissue cause localized perturbations in the magnetic field, and this is typically noted at the skull base adjacent to the sphenoid, frontal, and petrous air cells.

If regions of magnetic field heterogeneity are unwittingly included within the sampling voxel by the operator, MR spectroscopy may be suboptimal. Susceptibility-weighted imaging (SWI) is a relatively new imaging sequence that is increasingly routinely used. ${ }^{4}$ This sequence can detect subtle signal intensity phase differences within tissues, visualized as a signal intensity image or a phase map. ${ }^{4}$ Anatomic regions with increased susceptibility differences can contribute to magnetic field inhomogeneity. Therefore, SWI or phase maps could be used to guide placement of the MR spectroscopy sampling voxel or aid interpretation of poor spectral results.

\section{Technique}

MR imaging studies were performed at $1.5 \mathrm{~T}$ with use of a 12-channel receiver coil (Siemens, Erlangen, Germany). Data from a healthy subject and from a patient with recurrent glioblastoma multiforme were analyzed. We acquired SWI data by using a velocity-compensated radio-frequency-spoiled high-resolution $3 \mathrm{D}$ gradient-echo sequence

Received September 3, 2008; accepted after revision October 13.

From the Department of Medical Imaging (L.J., R.B., A.C.), Royal Brisbane and Women's Hospital, Brisbane, Queensland, Australia; Centre for Magnetic Resonance (A.A.S.) and Discipline of Medical Imaging (A.C.), University of Queensland, Brisbane, Australia.

Please address correspondence to Professor Alan Coulthard, Department of Medical Imaging, Level 3, Ned Hanlon Building, Royal Brisbane and Women's Hospital, Herston, QLD, Australia 4029; e-mail: a.coulthard@uq.edu.au

DOI 10.3174/ajnr.A1403
(TR, 49 ms; TE, 40 ms; 72 sections; matrix, 256.177; voxel size, $1.1 \times$ $0.9 \times 2 \mathrm{~mm}$ ). Phase and SWI maps were generated with use of VB15 software (Siemens). We acquired MR spectroscopy data by using a multivoxel point-resolved spectroscopy sequence (TR, $1700 \mathrm{~ms}$; TE, $135 \mathrm{~ms}$; MR spectroscopy voxel size, $10 \mathrm{~mm} \times 10 \mathrm{~mm} \times 15 \mathrm{~mm})$. Fully automated shimming and water suppression were used and peripheral saturation bands placed. A gadolinium-enhanced T1weighted acquisition (same section prescription as SWI) was used for placement of the multivoxel MR spectroscopy grid.

We investigated the relationship between spectral quality and the degree of susceptibility-induced phase distortion (ie, field inhomogeneity) by processing the phase maps into a $15-\mathrm{mm}$ maximum intensity projection section to match the MR spectroscopy voxel dimensions (Fig 1). For each individual MR spectroscopy voxel on the registered phase map, the averaged SD in phase was correlated with the spectral peak width at half-height for NAA, Cr, and Cho (Fig 2). Pearson correlation coefficients were determined to relate spectral quality (metabolite line width) with field inhomogeneity (magnitude of the susceptibility-induced phase change) for a particular spatial location. These coefficients were 0.56 for NAA, 0.60 for $\mathrm{Cr}$, and 0.60 for Cho $(P=.001)$.

\section{Discussion}

Magnetic field inhomogeneity identified on SWI-generated phase maps (indicated in Fig 2 by a increased SD of pixel values) correlates significantly with poor quality of spectroscopic data (widened metabolite peaks, poor water suppression, excessive baseline roll) obtained in both healthy tissue and contrast-enhancing tumor (Figs 1, 2, 3). Spectral quality may be improved by using the phase image to guide placement of the sampling voxel away from regions of poor field homogeneity in both single and multivoxel MR spectroscopy studies. In addition, voxel placement may be optimized by use of postcontrast SWI to identify areas of hemorrhage and contrast enhancement. Benefits of use of SWI data for MR spectroscopy voxel placement include higher-quality spectra, fewer repeated studies, faster study acquisition in critically ill and pediatric patients, and optimal use of scanner time.

\section{Acknowledgments}

We would like to acknowledge associate professor Stephen Rose for his assistance with this study. 


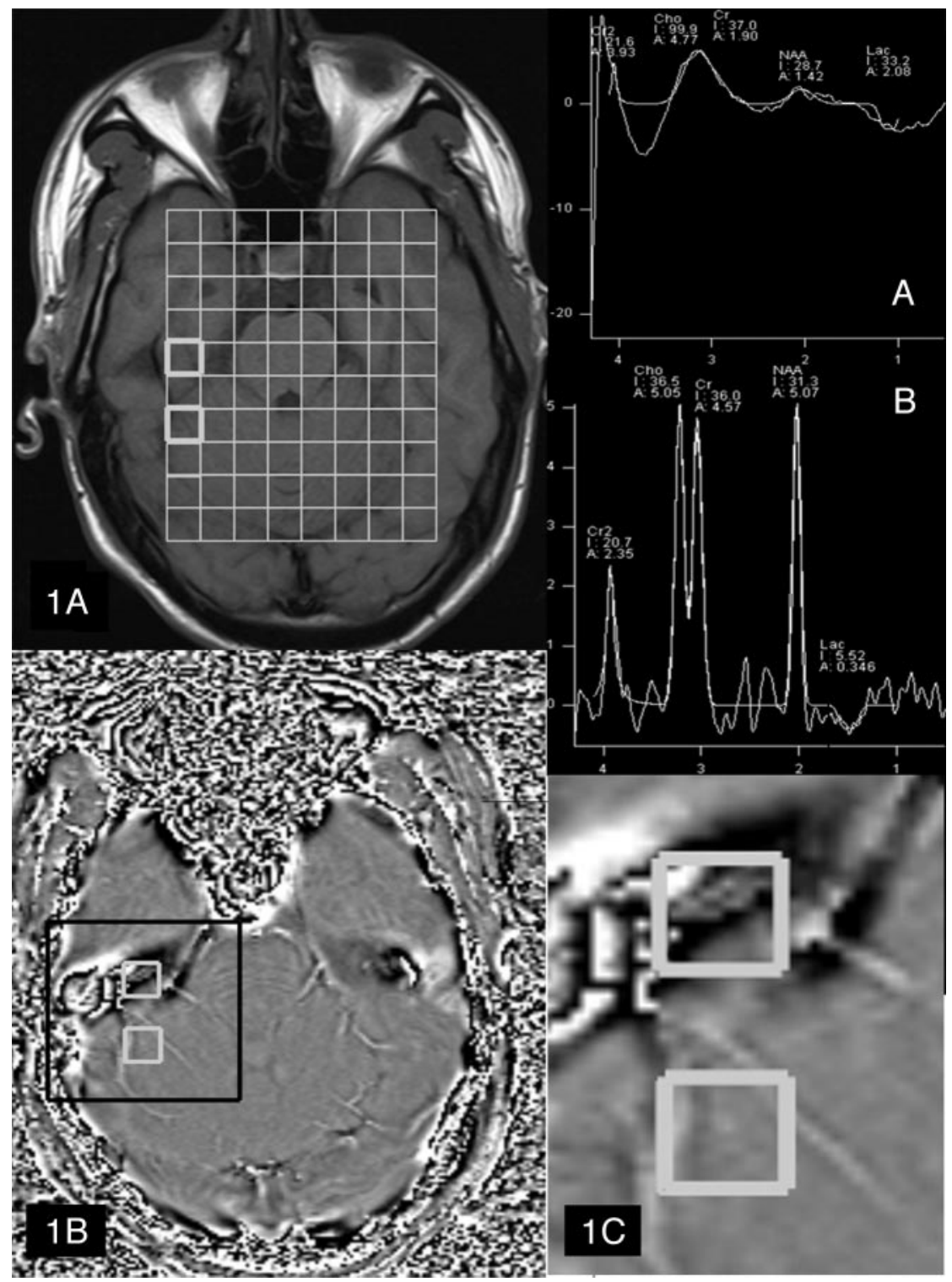

Fig 1. Representative data from a healthy subject. $1 A$ T1-weighted image showing placement of the multivoxe spectroscopy grid (top left) and (1B) SWI phase map Spectrum A for the anterior voxel corresponding to brain tissue exhibiting large, susceptibility-induced phase heterogeneity. This spectrum is of nondiagnostic quality. Spectrum $B$ is acquired from the more posterior voxel in which there is less phase shift compared with the anterior voxel and is of good diagnostic quality. $1 C$, Enlargement from $(1 B)$ showing increased phase heterogeneity within the anterior voxel.

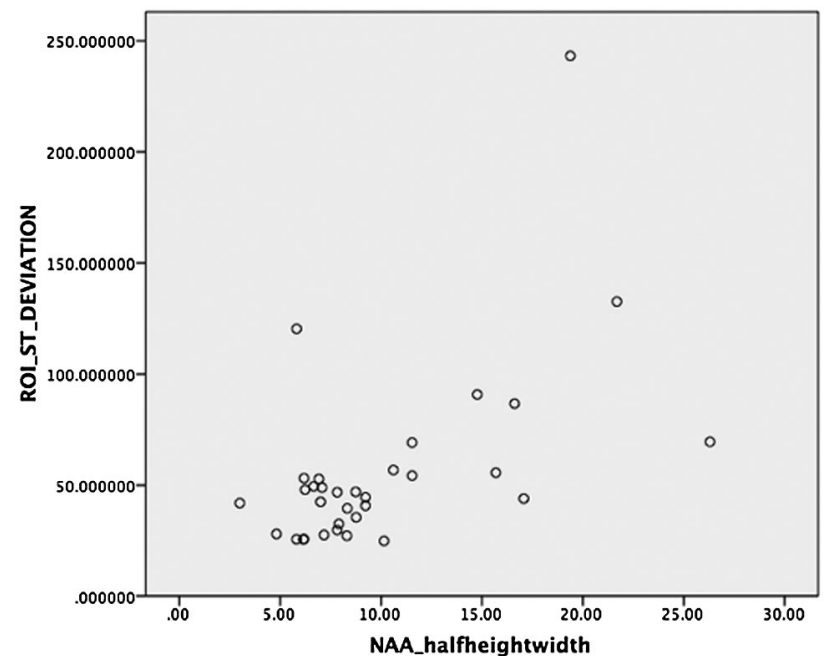

Fig 2. Relationship between spectral quality and phase heterogeneity. Correlation between the line width at half-height for NAA and pixel SD for each MR spectroscopy voxel within the phase image (Pearson rank correlation coefficient, 0.56; $P=.001$ ). 


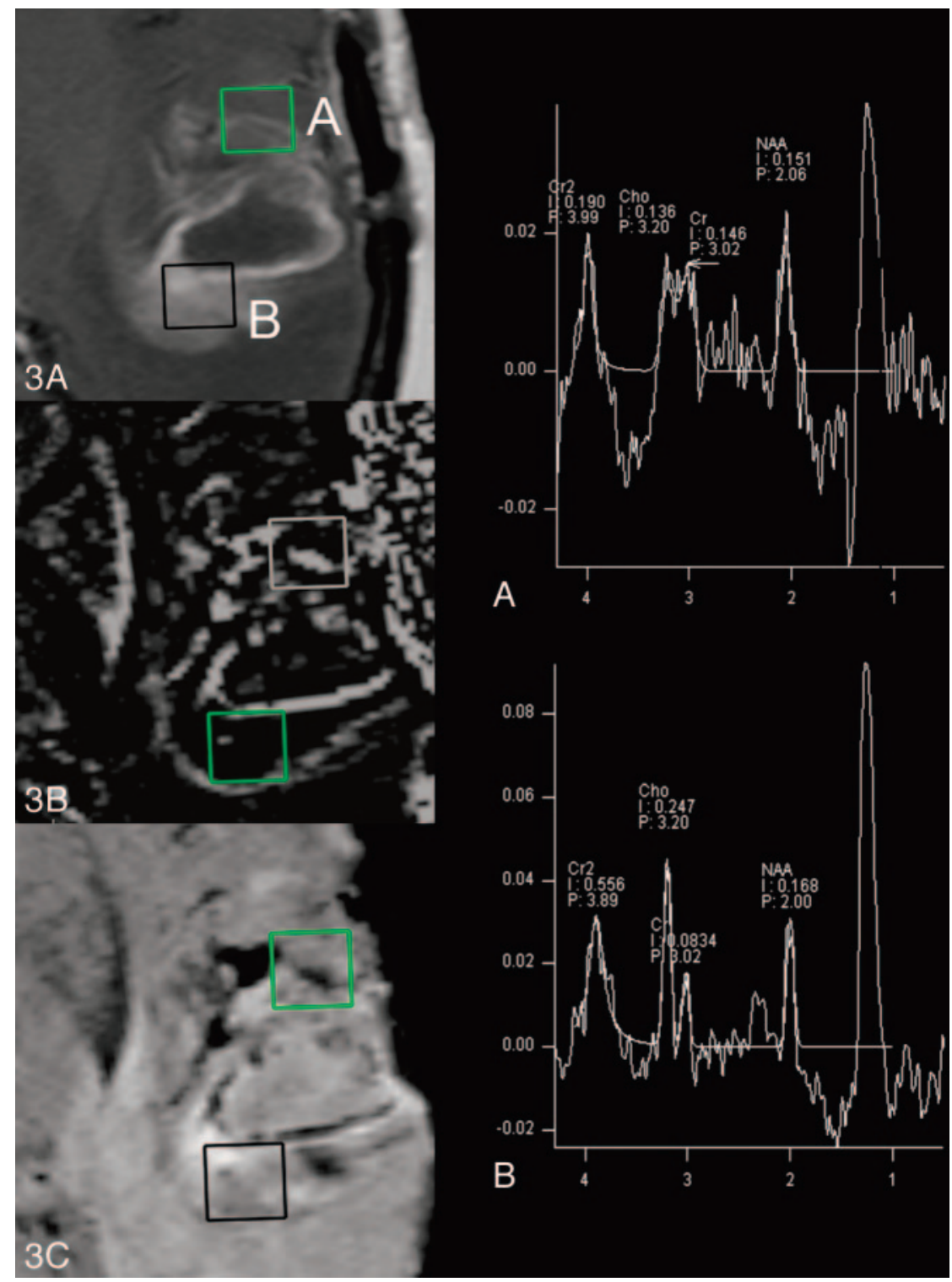

Fig 3. $3 A$, T1-weighted postgadolinium image showing tumor enhancement and 2 representative sampling voxels $A$ and $B$. Voxel $A$ is placed in an area correlating to magnetic field heterogeneity on the phase map image (3B). This heterogeneity is secondary to the paramagnetic hemorrhagic tissue seen on the SWI image (3C). The MR spectrum from voxel $A$ is shown top right. Voxel $B$ has been placed in a region without phase heterogeneity $(3 B)$ and with contrast enhancement $(3 A, 3 C)$ with resultant diagnostic spectrum $(B$, bottom right).

\section{References}

1. Lin A, Ross BD, Harris $\mathrm{K}$, et al. Efficacy of proton magnetic resonance spectroscopy in neurological diagnosis and neurotherapeutic decision making. NeuroRx 2005;2:197-214

2. Di Costanzo A, Trojsi F, Tosetti M, et al. High-field proton MRS of human brain. Eur J Radiol 2003;48:146-53
3. Drost DJ, Riddle WR, Clarke GD, et al. Proton magnetic resonance spectroscopy in the brain: report of AAPM MR Task Group \#9. Med Physics 2002;29:2177-97

4. Rauscher A, Sedlacik J, Barth M, et al. Magnetic susceptibility sensitive MR phase imaging of the human brain. AJNR Am J Neuroradiol 2005;26: $736-42$ 